\title{
Dialysis Access: Percutaneous Endovascular Interventions under Sonographic Guidance Alone
}

\author{
Daniel Périard ${ }^{1 *}$, Marie-Antoinette Rey Meyer ${ }^{1}$, Ould Mahoud Hemett ${ }^{2}$, Jean-Jacques Mottet ${ }^{2}$, Olivier Phan ${ }^{3,4}$, Rolf P Engelberger ${ }^{1,5}$ and Daniel \\ Hayoz $^{1}$
}

${ }^{1}$ Clinic of Angiology, HFR Hôpital Cantonal, Fribourg, Switzerland

${ }^{2}$ Clinic of Nephrology, HFR Hôpital Cantonal, Fribourg, Switzerland

${ }^{3}$ Hémodialysis, Hôpital Intercantonal de la Broye, Payerne, Switzerland

${ }^{4}$ Department of Nephrology, CHUV, Lausanne University Hospital, Switzerland

${ }^{5}$ Clinic for Angiology, Swiss Cardiovascular Center, Inselspital, University Hospital and University of Bern, Switzerland

\begin{abstract}
Purpose: Prosthetic shunts or native arteriovenous fistulas (AVFs) often require surgical or percutaneous endovascular interventions to correct stenosis or remove thrombosis. Catheterizations usually require radiation and iodinated contrast medium. Sonography and color-Doppler provide reliable images and measurement of dialysis access anatomy and volume flow, and have therefore a great potential to allow dialysis access percutaneous interventions in simple examination rooms without any contrast medium and any radiation, sparing time and human resources.
\end{abstract}

Methods: From September 2011 to June 2016, all patients with dialysis access requiring percutaneous angioplasty, stenting, or thrombectomy were included in this cohort study and treated under sonography guidance alone. Patients with target lesion in central vessels were excluded. Efficacy outcomes were defined as improvement in volume flow and peak systolic velocity (PSV) of the stenosis.

Results: Sixty-six procedures were performed in 31 patients $(21$ (66.7\%) males; $65.5 \pm 15.1$ years old). There was no adverse event except moderate pain during balloon angioplasty. Primary success of the procedure was $97.0 \%$. The volume flow rose from $449 \pm 241 \mathrm{~mL} / \mathrm{min}$ to $786 \pm 262 \mathrm{~mL} / \mathrm{min}$ and the PSV decrease from $6.1 \pm 0.9$ to $3.1 \pm 1.1 \mathrm{~m} / \mathrm{s}$. The median time to next intervention was 10.9 months with $82.9 \%$ of re-interventions performed under sonography guidance again.

Conclusions: Sonographic guidance alone is efficient and safe, for the majority of percutaneous interventions on prosthetic or native access. This approach is much simpler and cheaper, does not expose the patient and physician to radiations, and does not require contrast medium.

Keywords: Vascular access; Dialysis; Angioplasty; Ultrasoundguided; Ultrasound guidance; Thrombectomy

\section{Introduction}

Dialysis access often requires surgical or endovascular intervention to promote maturation, correct stenosis, avoids or manages acute occlusion. The development of dedicated catheters has allowed an increasing use of endovascular procedure to maintain prosthetic or native AVFs functional. Several reasons call for a shift from angiography suite to more simple office-based sonography-guided interventions: these procedures are usually straightforward, rapid, and the equipment is simple and predictable. The vessels to treat are superficial, allowing continuous sonographic assessment with high quality images. B-mode and duplex scan provide continuous accurate imaging of vessel wall, lumen size and volume flow estimation. The access site management is usually simple and much safer than in arterial procedures. Sonography guidance does not require fluoroscopy and therefore avoids patients and caregivers exposition to radiations. There is no need for contrast media avoiding their potential side effects. For the patients, procedures performed in the office are more comfortable and less stressful than in angiography suite and there is no lost time waiting for the cathlab vacancy. For the healthcare system, sonography-guided procedures are much less expensive requiring less equipment and only two persons for less than $60 \mathrm{~min}$ instead of 3 to 4 persons for longer.

Feasibility of the ultrasound guidance was already investigated in a few previous studies. Procedures were first performed in the cathlab under sonography guidance, limiting the use of fluoroscopy to bail-out procedure [1-6]. After exclusion of cases with central vein stenosis (where duplex scan does not provide sufficient image quality) three authors then investigated the feasibility of AVFs angioplasty or AVFs balloon-assisted maturation in an office-based setting with very encouraging results [1-3]. Overall these first studies demonstrated the feasibility of this technique without experiencing major adverse event [4-6]. Therefore, since this new approach seems very elegant, we designed this study to prospectively assess the efficacy and the safety of AVFs endovascular procedures in an office setting under sonography guidance alone, in a tertiary hospital.

\section{Subjects and Methods}

From September 2011 to June 2016, all consecutive patients with dialysis access requiring percutaneous angioplasty, stenting,

*Corresponding author: Daniel Périard, Clinic of Angiology, HFR Hôpital Cantonal, Fribourg, Switzerland, Tel: +41 2642672 52; E-mail: daniel.periard@h-fr.ch

Received February 10, 2017; Accepted February 19, 2017; Published February 24, 2017

Citation: Périard D, Meyer MAR, Hemett OM, Mottet JJ, Phan O, et al. (2017) Dialysis Access: Percutaneous Endovascular Interventions under Sonographic Guidance Alone. J Vasc Med Surg 5: 304. doi: 10.4172/2329-6925.1000304

Copyright: @ 2017 Périard D, et al. This is an open-access article distributed under the terms of the Creative Commons Attribution License, which permits unrestricted use, distribution, and reproduction in any medium, provided the original author and source are credited. 
or thrombectomy were included in this single arm cohort study. We included all patients with native or prosthetic AVFs requiring intervention on the arterial anastomosis, in the prosthesis or in the venous outflow. We included venous stenosis or occlusion up to the sub-clavian vein and excluded patients with known or suspected central vein stenosis.

All patients had a B-mode and duplex scan examination of their arm arteries, AVF and venous outflow up to the sub-clavian vein. The duplex scan-derived mean volume flow (VFs), the peak systolic velocity (PSV) of all stenosis and minimal and reference lumen diameter of each stenotic segment were measured before and at the end of the intervention. Estimates of VFs were based on the mean of at least two congruent measurements in a non-tortuous segment of the brachial artery or in the prosthesis. All interventions were performed with a linear $12 \mathrm{MHz}$ probe (Affiniti 70, Philips Medical Systems) [7]. The interventions were performed in a simple examination room in our outpatient clinic by an experimented angiologist assisted by one vascular nurse.

All patients gave their informed consent before the procedure. Once comfortably installed on the examination table, skins were disinfected using povidone-iodin and sterile drape were placed. After local anesthesia with $1 \mathrm{~mL}$ of lidocaine $1 \%$, a $4 \mathrm{~cm}$ long 6 french sheath (Prelude short, Merit Medical) was placed. Heparin 2500 Units was usually administered except for patients receiving vitamin $\mathrm{K}$ antagonists or for short interventions. Stenosis was usually crossed by an 80 or $120 \mathrm{~cm} 0.035$ inch guidewire and predilatation of the lesion was performed with $50 \mathrm{~cm}$ dedicated plain balloon catheter (Reef HP, Medtronic). Drug-eluting balloon angioplasty was then applied to almost all lesions, using either $80 \mathrm{~cm}$ or $40 \mathrm{~cm}$ long Freeway (Eurocor) or Admiral Impact (Medtronic) inflated to nominal pressure for at least $2 \mathrm{~min}$. Highly fibrotic lesions were predilated using multiple inflations with a $50 \mathrm{~cm}$ long cutting-balloon (Boston Scientific), until reaching satisfactory lumen. The balloon length $(20$ to $60 \mathrm{~mm})$ and diameter $(5$ to $8 \mathrm{~mm}$ ) were chosen based on vessel diameter measured at the edge of the lesion. All balloon catheters were inflated using saline without contrast media. In a few cases of persisting severe stenosis despite repeated angioplasties, a nitinol self-expandable stent was placed (Misago, Terumo). The puncture site was closed by light compression during a few minutes. Final VFs and PSVs were measured after sheath removal. Patients were allowed to leave the clinic immediately after the procedure.

The efficacy outcomes were defined as the increase in VFs of the fistula, the decrease in the PSVs of the most severe stenosis and the survival time without new AVF intervention. Safety outcome was defined as any adverse event related to the procedure and procedure failure defined by inability to perform the next dialysis without further intervention. All patients were followed at 3 months and then on an annual basis during the study period. Proportions were expressed as percentage and standard deviations. Statistical analyses were performed using Stata 9.

\section{Results}

During the study period, 74 AVFs endovascular procedure were considered for inclusion in 37 patients. For 8 procedures (6 patients) a previous central vein stenosis was known ( 3 cases) or suspected (5 cases) on the basis of proximal venous resistance to compression at duplex scan. These procedures were performed in angiography suit and excluded from the study. The 66 remaining procedures (31 patients) were performed under sonography alone, in a standard examination room. The clinical characteristic of the 31 patients included are shown in the Table 1 . The patients ( $66.7 \%$ males; $65.5 \pm 15.1$ years old) were on hemodialysis since $27 \pm 30$ months. The AVFs were native radiocephalic fistulas in $73.3 \%$ and prosthetic forearm graft in $16.6 \%$.

\section{Interventions}

The indications for interventions were reduced flow in $66.7 \%$, fistula salvage for near occlusion or occlusion in $13.6 \%$, and arm edema or prolonged bleeding at needle retrieval in $9.1 \%$ (Table 2). We treated $1.5 \pm 0.7$ ( 1 to 3 ) stenosis per patient, in the outflow vein $(78.8 \%)$, in the prosthetic graft $(19.7 \%)$ or in the brachial artery $(1.5 \%)$. Plain balloon $(39.4 \%)$ or cutting balloon $(16.7 \%)$ was completed by drugeluting balloon angioplasty in $72.7 \%$. Thrombectomy of an acute AVF occlusion was successfully performed with a Rotarex 6 french device (Straub Medical) before angioplasty of the culprit stenosis.

\section{Efficacy and safety}

Primary success of the procedure was $97.0 \%$ (Table 3). The procedure failed to restore functional AVFs in two patients (one with a non-maturating radiocaphalic native fistula and one patient with an immediate reocclusion of the arm basilica vein by elastic recoil). The mean PSV of the worst stenosis decreased from $6.1 \pm 0.9$ to $3.1 \pm$ $1.1 \mathrm{~m} / \mathrm{s}$. The volume flow rose from $449 \pm 241$ to $786 \pm 262 \mathrm{~mL} / \mathrm{min}$ The intervention was well tolerated without any severe or significant

\begin{tabular}{|l|c|}
\hline & Patients $\mathbf{n = 3 1}$ \\
\hline Age (years) & $65.4 \pm 12.0$ \\
\hline Gender (male) & $21(67.7 \%)$ \\
\hline Diabetes & $38.7 \pm 49.5 \%$ \\
\hline Hypertension & $77.4 \pm 42.5 \%$ \\
\hline Smoking (past or current) & $48.4 \pm 50.8 \%$ \\
\hline Hyperlipidemia & $61.3 \pm 49.5 \%$ \\
\hline Dialysis duration (months) & $27 \pm 29$ \\
\hline Type of AV access & \\
\hline$\cdot \quad$ Native radio-cephalic & $71.0 \%$ \\
\hline$\cdot \quad$ Prothetic forearm & $18.2 \%$ \\
\hline$\cdot \quad$ Prothetic radio-cephalic & $3.2 \%$ \\
\hline$\quad \quad$ Native brachio-basilic & $3.2 \%$ \\
\hline$\quad$ Prothetic arm & $3.2 \%$ \\
\hline
\end{tabular}

Table 1: Patients baseline characteristics.

\begin{tabular}{|c|c|c|}
\hline & & Interventions $\mathrm{n}=66$ \\
\hline \multicolumn{3}{|c|}{ Indication } \\
\hline • & Low flow & $66.7 \%$ \\
\hline • & Salvage (near occlusion or occlusion) & $13.6 \%$ \\
\hline • & Prolonged bleeding at needle retrival & $9.1 \%$ \\
\hline - & Favor vein maturation & $4.5 \%$ \\
\hline • & Aneurysm or pseudo-aneurysm & $3.0 \%$ \\
\hline • & Hand ischemia & $1.5 \%$ \\
\hline nb Sten & s treated per patient & $1.5 \pm 0.7$ \\
\hline \multicolumn{3}{|c|}{ Endovascular intervention } \\
\hline - & Plain balloon angioplasty & $39.4 \%$ \\
\hline - & Drug eluting balloon angioplasty & $72.7 \%$ \\
\hline • & Cutting or scoring balloon angioplasty & $16.7 \%$ \\
\hline • & Stenting & $6.1 \%$ \\
\hline - & Thrombectomy & $1.5 \%$ \\
\hline \multicolumn{3}{|c|}{ Site of intervention } \\
\hline - & Vein & $78.8 \%$ \\
\hline - & Prosthesis & $19.7 \%$ \\
\hline - & Artery & $1.5 \%$ \\
\hline
\end{tabular}

Table 2: Interventions characteristics. 
adverse event. There was no vein rupture and no local hematoma. Local pain was sometimes observed during balloon inflations. However this was limited to the inflation time. In 3 procedures, local anesthesia was injected next to the lesion to allow sufficient balloon inflation. No patient required any sedation or general anesthesia. The mean intervention time of the last 30 procedures was 21 minutes from sheath insertion to sheath removal. The median duration up to the next AVF intervention was 10.9 months. During the study period 35 re-interventions were necessary, of which $82.9 \%$ were again performed under sonography alone (Table 3).

The subgroup of 11 interventions on thigh fibrotic stenosis performed with cutting- and drug eluting balloon provided satisfying results. In this group the intervention was a success for all and the lower preprocedural VF due to the severity of the lesion rose from 383 \pm 134 to $713 \pm 176 \mathrm{~mL} / \mathrm{min}$.

\section{Discussion}

This study shows that ultrasound guidance is a safe technique and provides an excellent monitoring during AVFs/AVGs percutaneous interventions, with satisfying clinical results. The primary success was achieved for all but two patients. These two failures occurred at the beginning of the study when experience was still to be acquired. During the study we observed a progressive reduction of the intervention duration, achieving very short intervention time from sheath insertion to sheath removal and less than one hour duration from door to door. This progression was due to the gain in technical experience and the development of shorter dedicated material. This allowed a progressive reduction in the dose of heparin administered, allowing some procedures to be done without antithrombotic drug, with very short compression time after sheath retrieval. These aspects were particularly appreciated by the patients.

A major impact in this field is the absence of iodinated contrast media for US-guided procedure. Although renal function is already lost, contrast mediums may provoke fluid overload, heart failure, allergies and intolerance. The absence of fluoroscopy in this new technique is a benefit for the patients who are frequently exposed to radiation but also for the interventionists whose hands are usually highly exposed during AVFs interventions. Besides the absence of radiation, ultrasound-guided interventions does not require heavy radio-protective jacket and can be performed comfortably seated. Most importantly, the reduced need in personal ( 2 persons) for this intervention, and the reduced need in technical equipment make this new technique much cheaper and practicable in office, dialysis center or clinics without heavy equipment.

\begin{tabular}{|l|c|}
\hline Results & Interventions $\mathbf{n = 6 6}$ \\
\hline Primary success of the intervention* & $97.0 \%$ \\
\hline Vol flow before $(\mathrm{mL} / \mathrm{min})$ & $449 \pm 241$ \\
\hline Vol flow after $(\mathrm{mL} / \mathrm{min})$ & $786 \pm 262$ \\
\hline PSV at worst stenosis before $(\mathrm{m} / \mathrm{s})$ & $6.1 \pm 0.9$ \\
\hline PSV at worst stenosis after $(\mathrm{m} / \mathrm{s})$ & $3.1 \pm 1.1$ \\
\hline Mean time to next intervention $(\mathrm{m})$ & 12.1 \\
\hline Median time to next intervention $(\mathrm{m})$ & 10.9 \\
\hline Next intervention & $\mathbf{n = 3 5}$ \\
\hline$\cdot \quad$ US-guided & $82.9 \%$ \\
\hline$\cdot \quad$ Fluoroscopy-guided & $5.7 \%$ \\
\hline$\quad$ Surgical correction & $8.6 \%$ \\
\hline$\quad$ Surgical confection of new access & $2.9 \%$ \\
\hline
\end{tabular}

*Next dialysis possible without further intervention.

Table 3: Efficacy and safety outcomes.
A concern could be the lack of central vein examination during US-guided procedure, with the potential risk of incomplete procedure leaving untreated central vein stenosis, whereas central vein are routinely examined during phlebography in cathlab. Despite this lack of direct visualization, a systematic examination of venous outflow (including appreciation of the vein tension by compression) is usually sufficient to suspect a central vein stenosis responsible of a resistance on central venous return. This may be overcome by a careful duplex examination before any procedure. In our study, 5 patients were excluded for suspected central vein stenosis at duplex screening and underwent a conventional phlebography that confirmed the stenosis. Among the 66 patients treated under US-guidance, only 2 patients required a central vein phlebography during follow-up, due to prolonged bleeding time at needle retrieval despite good results after peripheral intervention. Therefore we believe that despite these two re-interventions, the overall benefit for this population remained important.

In this study, interventions were performed by trained angiologists with a large experience of duplex AVFs examination and peripheral artery or vein catheterization. We observed no adverse event related to the procedure, a result consistent with the very few complication rates in the previously published series. In their series of 32 office-based ultrasound-guided interventions, Ascher et al. had one vein rupture and one vein dissection that could be managed conservatively. They achieved an increase in blood flow from 350 to $933 \mathrm{~mL} / \mathrm{min}$, a larger increase than in our study [4]. These differences may be due to larger diameters achieved by PTA or by inclusion of a higher proportion of non-maturating AVFs at higher risk of vein rupture. This is also the case in the study of Gorin et al., who experienced a complication rate of $11 \%$. In their study, 48 of the 55 procedures were performed on non-maturating AVFs [6]. In our study, we mostly treated de novo stenosis of maturated AVFs. Vein were dilated at their reference diameter without aiming oversizing. This probably account for the absence of vein rupture. Our lower post-procedural VFs $(786 \mathrm{~mL} /$ $\mathrm{min}$ ), is probably explained by a majority of native less than 3 years old radio-cephalic AVFs. However the satisfying survival time to next intervention support this careful protocol and we therefore recommend a precise calibration of vessel lumen adjacent to the stenosis before each angioplasty and careful interventions on non-maturating AVFs.

In conclusion, this study confirms that office-based endovascular repair of AVFs under sonography-guidance is as safe, as efficient, simpler, shorter, more comfortable and cheaper than under fluoroscopic guidance.

\section{Conflicts of Interest}

The authors declare no conflict of interest regarding this study.

\section{References}

1. Wakabayashi M, Hanada S, Nakano H, Wakabayashi T (2013) Ultrasoundguided endovascular treatment for vascular access malfunction: results in 4896 cases. J Vasc Access 14: 225-230.

2. Bojakowski K, Góra R, Szewczyk D, Andziak P (2013) Ultrasound-guided angioplasty of dialysis fistula - technique description. Pol J Radiol 78: 56-61.

3. Liang HL, Fu JH, Wang PC, Chen MC, Wang CC, et al. (2014) Endovascular salvage of immature autogenous hemodialysis fistulas. Cardiovasc Intervent Radiol 37: 671-678.

4. Ascher E, Hingorani A, Marks N (2009) Duplex-guided balloon angioplasty of failing or nonmaturing arterio-venous fistulae for hemodialysis: a new officebased procedure. J Vasc Surg 50: 594-599.

5. Gallagher JJ, Boniscavage P, Ascher E, Hingorani A, Marks N, et al. (2012) Clinical experience with office-based duplex-guided balloon-assisted maturation of arteriovenous fistulas for hemodialysis. Ann Vasc Surg 26: 982-984. 
Citation: Périard D, Meyer MAR, Hemett OM, Mottet JJ, Phan O, et al. (2017) Dialysis Access: Percutaneous Endovascular Interventions under Sonographic Guidance Alone. J Vasc Med Surg 5: 304. doi: 10.4172/2329-6925.1000304

Page 4 of 4

6. Gorin DR, Perrino L, Potter DM, Ali TZ (2012) Ultrasound-guided angioplasty of autogenous arteriovenous fistulas in the office setting. J Vasc Surg 55: 1701-1705.
7. Ascher E, Hingorani A, Marks N (2010) Duplex scanning-derived access volume flow: novel predictor of success following endovascular repair of failing or nonmaturing arteriovenous fistulae for hemodialysis. Vascular 18: 9-13. 